\title{
Atmospheric Oxidation of NbTi Superconductor
}

\author{
T Davies ${ }^{\mathrm{a}}$, C R M Grovenor ${ }^{\mathrm{a}}$, S C Speller ${ }^{\mathrm{a}, *}$ \\ ${ }^{a}$ Department of Materials, University of Oxford, Parks Road, OX1 3PH, UK
}

\begin{abstract}
$\mathrm{Nb}-47 \mathrm{wt} \% \mathrm{Ti}$ is the workhorse alloy of the superconducting magnet industry. Fabrication of "perfect" superconducting joints with electrical resistance values $<10^{-12} \Omega$ is necessary to operate the magnets in persistent-mode without a power supply, enabling the generation of the high stability magnetic fields required for MRI and NMR applications. Many jointing techniques involve exposure of the highly-reactive metallic filaments to air, and it is well known that exposures of more than a few minutes prevent successful superconducting joint formation. Here we present the first fundamental study of the oxidation kinetics and chemistry of the oxide layers on a superconducting NbTi alloy. We use angle-resolved x-ray photoelectron spectroscopy (AR-XPS) to analyse both the earliest stages of oxidation by gas dosing with pure oxygen in UHV, and also oxidation under industrially relevant conditions by exposure of clean surfaces to laboratory air for timescales up to an hour. The reaction layer is found to be a mixed $\mathrm{Nb} / \mathrm{Ti}$ oxide, with little change in the Nb:Ti ratio with depth. The oxide formation follows logarithmic growth kinetics, increasing to a limiting thickness of $3.7 \pm 0.3 \mathrm{~nm}$ after extended exposure to air.
\end{abstract}

Keywords: Superconductor, AR-XPS

\section{Introduction}

Niobium-47wt\% titanium ( $\mathrm{NbTi}$ ) is the most commercially important superconducting material, with Magnetic Resonance Imaging (MRI) magnets based

\footnotetext{
* Corresponding author

Email address: susannah.speller@materials.ox.ac.uk (S C Speller)
}

Preprint submitted to Journal of Alloys and Compounds

June 29, 2020 
on NbTi making up over $80 \%$ of the global superconductivity market 1 . Whilst

${ }_{5}$ other materials provide superior superconducting transition temperatures $\left(\mathrm{T}_{c}\right)$ and upper critical fields $\left(\mathrm{B}_{c 2}\right)$, none can rival the high ductility, ease of processing and low price of NbTi which make it ideal for mass-production of superconducting magnets [2]. Formation of truly superconducting (persistent) joints is essential in meeting the extremely stringent field stability requirements of MRI magnets [3], and is complicated by the wire geometry in which fine NbTi filaments are enclosed in a copper matrix. There is a well-known solder technique for joint fabrication in which exposure of the NbTi filaments to air can be largely avoided, the matrix-replacement method [4, but this is not universally applicable to all the NbTi wires currently used in the magnet industry [5]. Alternative 15 jointing techniques such as cold-pressing are also being explored because they do not rely on the use of toxic lead-based solders which are becoming subject to strict legislation [6, 7]. Practical deployment of these solder-free techniques on the factory floor will inevitably involve exposing the NbTi to air for short periods [8, 9, 3, 10]. Since even a thin (few nm) non-superconducting oxide layer will introduce unacceptable resistance into the circuit, it is crucial to understand the fundamental oxidation behaviour of NbTi to enable reliable persistent joint production processes to be designed.

$\mathrm{X}$-ray photoelectron spectroscopy (XPS) is a powerful technique for the analysis of the formation of thin oxide layers due to the small sampling depth and 25 good chemical sensitivity. In particular, angle-resolved XPS (AR-XPS) allows the depth distribution of different chemical species within the top few nanometres of the surface to be determined [11, and this has been utilised to study the oxidation of a range of material systems under ambient conditions, including magnesium [12, aluminium [13] and iron [14. AR-XPS studies have also been undertaken on niobium [15, 16, 17, 18] and titanium [19, 20] oxidised under ambient conditions, revealing both to have a distinct layered structure with the main stable oxide $\left(\mathrm{Nb}_{2} \mathrm{O}_{5}\right.$ and $\mathrm{TiO}_{2}$ respectively) forming on top of a series of suboxides. However, despite the relevance for jointing processes described above, no previous studies have investigated the oxidation of the Nb-47wt\% $\mathrm{Ti}$ 
superconducting alloy under ambient conditions.

\section{Analysing XPS data from thin oxide films}

XPS is based on the photoelectric effect, where photons incident on a material are absorbed and electrons are emitted with a particular kinetic energy dependent on the frequency of the incident light [21. The detected intensity of

40 photoelectrons originating from a buried layer of thickness $\delta z$ decreases exponentially with depth $d[1]$, given by

$$
\delta I=\sigma T(E) A N(z) \exp \left[\frac{-z}{\lambda \cos \theta}\right] \delta z
$$

where $N(z)$ is the atomic concentration of the species belonging to a specific peak at depth z, $\sigma$ is the Scofield photoemission cross-section [22, $T(E)$ the analyser transmission function, $A$ the photoelectron emissivity area and $\theta$ the angle between the detector and the surface normal. The analyser transmission function and photoemission emissivity area are constants that are specific to the instrument and experimental set-up. In laboratory based XPS analysis, the angle and depth independent terms $(\sigma, A$ and $T(E))$ are typically combined to give a sensitivity factor, $S$, for a particular elemental peak. If multiple elements are present in a sample, the concentration of a particular element, $x_{a}$, in the sampled depth is given by

$$
x_{a}=\frac{I_{a} / S_{a}}{\sum_{n} I_{n} / S_{n}}
$$

A simple model of oxide growth on a metallic substrate assumes a homogeneous overlayer of oxide of thickness $t_{o x}$ above a metallic bulk [23, 24, 25]. Integrating Equation 1 over the two layers gives the total intensity of photoelectrons released from the bulk metal $\left(I_{m e t}\right)$ and oxide layer $\left(I_{o x}\right)$ as

$$
I_{m e t}=\lambda_{m e t} N_{m e t} \sigma T(E) A \cos \theta \exp \left(\frac{-t_{o x}}{\lambda_{o x} \cos \theta}\right)
$$




$$
I_{o x}=\lambda_{o x} N_{o x} \sigma T(E) A \cos \theta\left[1-\exp \left(\frac{-t_{o x}}{\lambda_{o x} \cos \theta}\right)\right]
$$

Crucially, when comparing the peaks from the same element and electronic transition with just a small chemical shift resulting from differences in oxidation state, any variations in the cross section and instrumental factors are negligible. Therefore, accurate measurements of oxide thickness can be obtained using the well-known Strohmeier equation [24] based on ratios of the metal and oxide peak intensities that do not depend on instrumental factors.

$$
t_{o x}=\lambda_{o x} \cos \theta \ln \left[1+\frac{\lambda_{m e t} N_{m e t}}{\lambda_{o x} N_{o x}} \frac{I_{o x}}{I_{m e t}}\right]
$$

\section{Experimental Methods}

\subsection{Samples}

XPS experiments typically require a reasonably large area of flat surface. Commercial NbTi wire is not suitable because it typically contains $<100 \mu \mathrm{m}$ diameter NbTi filaments embedded in a copper matrix. Here all measurements were performed on a sheet of NbTi sourced from Siemens Healthineers. This was produced from a large NbTi monocore strand that was extracted during the early stages of wire manufacture, before most of the thermomechanical process-

70 ing stages. This monolith was instead rolled to form a flat sheet, approximately $15 \mathrm{~mm} \times 0.5 \mathrm{~mm}$ in cross section. This was still clad in copper and had a niobium diffusion barrier layer between the NbTi and the $\mathrm{Cu}$ sheath.

Energy dispersive x-ray (EDX) measurements of many regions of the sheet confirmed the bulk composition of the sheet to be $\mathrm{Nb}-45.3 \pm 0.3 \mathrm{wt} \% \mathrm{Ti}$ (61 at\% $\mathrm{Ti}$ ). 75 X-ray diffraction (XRD) scans shown in Figure 1 show the sample to be a single phase, body-centred cubic $\beta-\mathrm{Nb}(\mathrm{Ti})$ solid solution. This differs from the final microstructure in NbTi wires, where $\alpha$-titanium nanoprecipitates are present. Whilst these precipitates are thermodynamically favourable below 600 ${ }^{\circ} \mathrm{C}$, kinetic factors prevent their formation without heavy deformation and long

80 heat treatments 26]. To explore whether this different microstructure in the 
as-received sheet NbTi affects the oxidation process, some samples were heat treated for 60 hours at $420{ }^{\circ} \mathrm{C}$ to induce nanoscale precipitation of $\alpha$-Ti, as confirmed by XRD analysis (Figure 1). These precipitates are much smaller than the XPS spot size (>30 microns) so the analysed regions contained large numbers of precipitates and will be representative of the two phase sample surface. Figure 2 compares $\mathrm{Nb} 3 \mathrm{~d}$ and Ti 2p XPS spectra from the as-received and heat-treated samples after exposure to 10,000 L of oxygen. Since there are no apparent differences in the spectra, the remaining experiments were carried out on the as-received, single phase NbTi sheet.

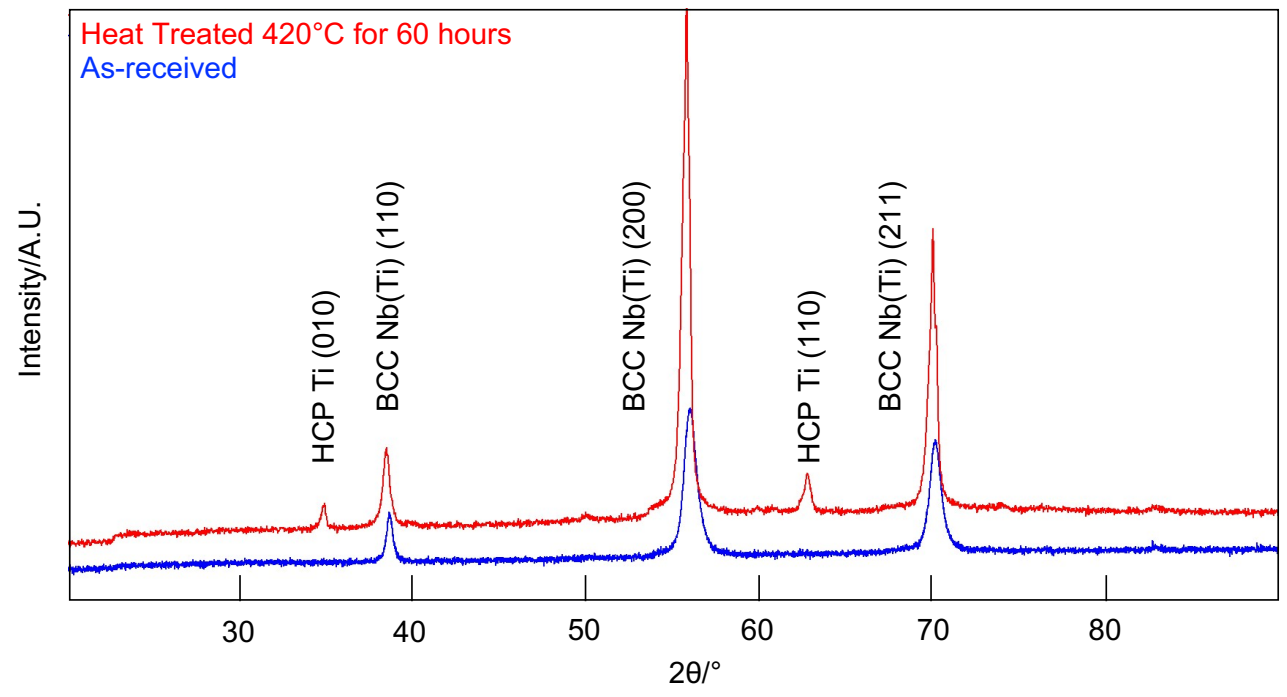

Figure 1: XRD scans of the NbTi sheet as-received and after heat treatment at $420{ }^{\circ} \mathrm{C}$ for 60 hours. The heat treated sample has extra peaks corresponding to the presence of $\alpha$-Ti precipitates.

\subsection{Surface Preparation}

The copper outer layer was first removed by etching in $30 \% \mathrm{HNO}_{3}: \mathrm{H}_{2} \mathrm{O}$ and the surface was ground to remove the niobium barrier layer. The samples were then polished with aqueous diamond suspension with particle sizes 6,3 and 1 microns to give a flat surface. Finally, the samples were cleaned in an ultrasonic 

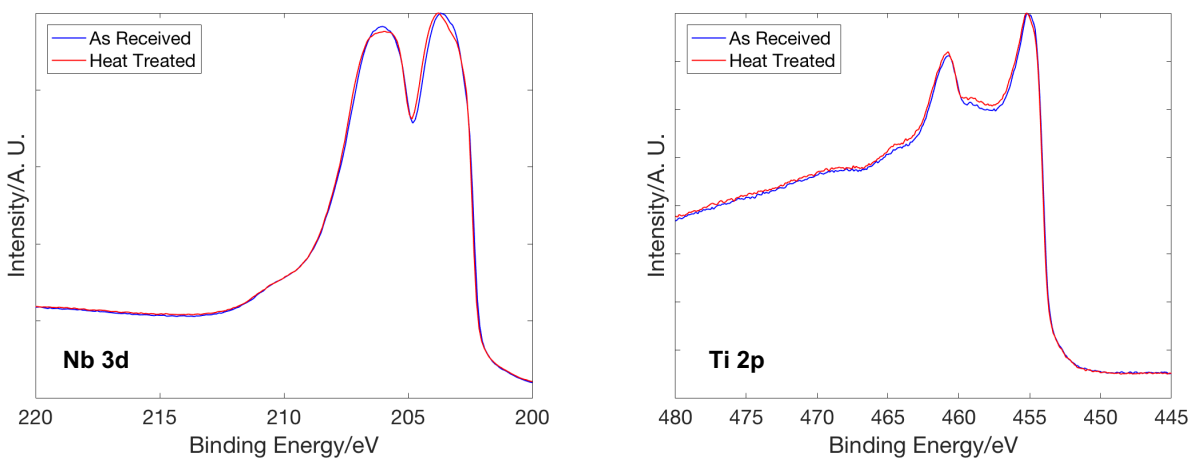

Figure 2: Nb and Ti normalised XPS spectra showing the similarity between as-received and heat-treated NbTi sheet.

bath for 10 minutes each in acetone and water to remove any contaminants (see supplementary information [27]). Sputter etching with argon ions was performed within the XPS instruments to remove any native oxide layer and obtain reproducible initial surface conditions for the oxidation experiments.

\subsection{X-ray Photoemission Spectroscopy Experiments}

100

Ambient air oxidations were performed and measured using a ThermoScientific K-alpha ${ }^{+}$instrument with monochromated Al K $\alpha$ x-ray source $(h \nu=1486.6$ $\mathrm{eV}$ ) and base pressure of approximately $10^{-7}$ mbar. Survey spectra were collected with a pass energy of $100 \mathrm{eV}$ averaged over four scans, whilst detailed spectra were collected with a pass energy of $20 \mathrm{eV}$ averaged over 10 scans. Oxidation was performed in the load-lock by exposing the sample to laboratory air.

AR-XPS analysis of samples after gas dosing in UHV was performed in a Kratos Axis Ultra spectrometer with delay line detector (DLD), monochromated Al K $\alpha$ x-ray source $(h \nu=1486.6 \mathrm{eV})$ and a base pressure of approximately $10^{-9}$ mbar. Survey spectra were collected with a pass energy of $160 \mathrm{eV}$ whilst detailed spectra were collected with a pass energy of $40 \mathrm{eV}$. The detector was operated in the hybrid mode, and the analysis area covered approximately 700 x 300 microns. Oxidation in this system was performed by admitting controlled 
amounts of pure, dry oxygen into a preparation chamber through a needle valve.

Both these spectrometers were fitted with tilt-stages to enable angle-resolved measurements.

In addition, some gas dosing experiments were carried out on the I-09 beamline at Diamond Light Source using $999.64 \mathrm{eV}$ "soft" x-rays and $5934.15 \mathrm{keV}$ "hard" x-rays using a VG Scienta EW4000 spectrometer with a wide collection angle up to $60^{\circ}$ and a spot size of approximately $300 \times 300$ microns.

\section{Experimental Results}

\subsection{Peak models}

Peak models have been developed to allow consistent fitting of Ti $2 \mathrm{p}$ and $\mathrm{Nb}$ 3d XPS spectra from samples prepared in different ways. Since each spectrometer has different instrumental broadening, separate models have been developed for each instrument. Peak fitting has been implemented in CasaXPS [28] using a Tougaard-type background 29] with symmetric peaks fitted using a standard Gaussian/Lorentzian blend with 30\% Lorentzian contribution, referred to as GL(30). The Metallic $\operatorname{Ti}(0)$ and $\mathrm{Nb}(0)$ peaks are typically asymmetric as a result of well-understood shake-up events [30. Therefore, these have been fitted using using the asymmetric Lorentzian (LA) peak option in CasaXPS which is modelled by

$$
L A(x: \alpha, \beta, F, E)= \begin{cases}L(x: F, E)^{\alpha} & : x \leq E \\ L(x: F, E)^{\beta} & : x>E\end{cases}
$$

where $\alpha$ and $\beta$ are parameters selected to add the desired asymmetry. $L(x$ : $F, E)$ is a Lorentzian with FWHM $F$ and maximum at binding energy $E$. The lineshape is entered in the form $\operatorname{LA}(\alpha, \beta, \mathrm{m})$ where $m$ defines the width of a Gaussian to convolve with the LA form.

\subsubsection{Titanium}

Titanium has three main stable oxides, $\mathrm{TiO}, \mathrm{Ti}_{2} \mathrm{O}_{3}$ and $\mathrm{TiO}_{2}$ [31, 32, that correspond to the $\mathrm{Ti}(\mathrm{II}), \mathrm{Ti}(\mathrm{III})$ and $\mathrm{Ti}(\mathrm{IV})$ oxidation states, respectively. The 
Ti $2 p$ transitions form a doublet due to spin orbit splitting of the $j=3 / 2$ and $\mathrm{j}=1 / 2$ states. Figure 3 compares typical Ti $2 \mathrm{p}$ spectra from sputter-cleaned and heavily oxidised surfaces. After the sputter etch, the spectrum is dominated by a doublet at binding energies characteristic of unoxidised metallic Ti. This peak is strongly asymmetric, with a spin-orbit splitting of approximately $6.1 \mathrm{eV}$. The asymmetry has been fitted using an $\operatorname{LA}(1.1,5,7)$ peak shape as suggested by Biesinger 33. The as-received surface demonstrates only a very small contribution from the metal, with the dominant feature being the $\mathrm{Ti}(\mathrm{IV})$ doublet at higher binding energy, corresponding to $\mathrm{TiO}_{2}$. Interestingly the $\mathrm{Ti}(\mathrm{IV}) 2 \mathrm{p}_{1 / 2}$ peak appears notably shorter and wider than the $\mathrm{Ti}(\mathrm{IV}) 2 \mathrm{p}_{3 / 2}$ peak. This is commonly attributed to the Coster-Kronig effect where the $1 / 2$ spin state is less stable than the $3 / 2$ state [34], leading to peak broadening. For detailed fits this was accounted for by allowing the full-width at half-maximum (FWHM) of the two peaks in the doublet to differ from one another, whilst maintaining the theoretical statistical area ratio of $2: 1$. The $\operatorname{Ti}(0) 2 \mathrm{p}_{1 / 2}$ and $\operatorname{Ti}(\mathrm{IV}) 2 \mathrm{p}_{3 / 2}$ peaks occur at approximately the same binding energy, leading to peak overlaps when both species are present, and hence careful fitting of the peaks is required to deconvolve the two contributions.

Both the sputter-cleaned and fully oxidised samples demonstrate an extra feature at high binding energies, $\approx 467 \mathrm{eV}$ in the sputter cleaned sample and $\approx 472 \mathrm{eV}$ in the oxidised sample. Measurements on a pure Nb standard sample have confirmed that this feature originates from the $\mathrm{Nb} 3$ s transition [27].

Samples oxidised for an intermediate amount of time demonstrate a signal that is a convolution of these two extreme cases. This is clear in the titanium 2p spectrum shown in Figure 4 for a NbTi sheet oxidised for one minute in laboratory air. Two extra doublets are required for a good fit, corresponding to the contributions from $\mathrm{Ti}(\mathrm{II})$ and $\mathrm{Ti}(\mathrm{III})$ oxides. Each oxidation state has been fitted with a doublet matching the theoretical 2:1 area ratio, after Biesinger et al. 33. The binding energies and FWHM values extracted from the fits are detailed in Table 1. The two peaks corresponding to different spin states in the $\operatorname{Ti}(0)$ and $\mathrm{Ti}(\mathrm{IV})$ doublets were allowed to have different FWHM values to account 

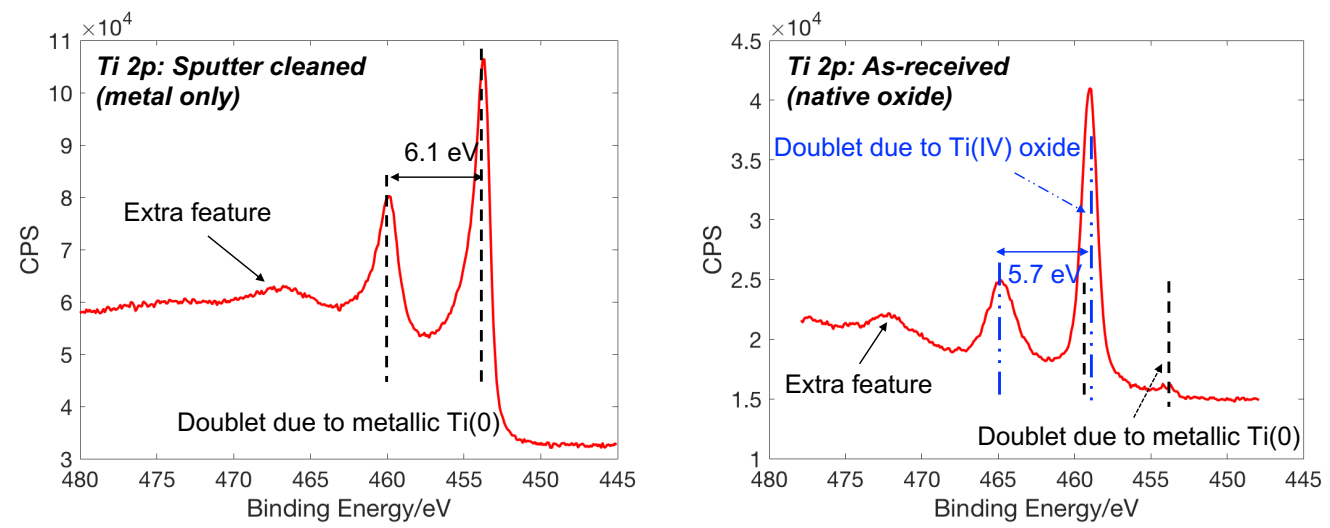

Figure 3: Left: Detailed scan of the titanium 2p region of a sputter cleaned NbTi sample, taken on the ThermoScientific K-Alpha ${ }^{+}$spectrometer parallel to the surface normal. The metallic doublet demonstrates strong asymmetry. Right: Detailed scan of the titanium $2 \mathrm{p}$ region of an as-received NbTi sample taken on the Kratos Axis Ultra spectrometer parallel to the surface normal. Both scans show one very strong doublet, although detailed fitting shows a small contribution from other oxidation states (this is discussed in detail for the as-received sample in Figure 13 and for the general case in Figure 44. In each case, there is an extra feature, the details of which will be discussed in the main text

for the Coster-Kronig effect, whilst the peaks in the Ti(II) and $\mathrm{Ti}(\mathrm{III})$ doublets were fixed to have the same FWHM value. Unlike Biesinger, the FWHM of these oxidation states were allowed to differ, although we note the similarity in the final fitted values. All peaks were fitted with a GL(30) peak shape apart from the $\operatorname{Ti}(0)$ peaks, which were fitted with an asymmetric $\mathrm{LA}(1.1,5,7)$ peak shape. The extra Nb 3s contributions were accounted for by adding two extra singlet peaks using symmetric GL(30) line shapes. To transfer this model to other spectra collected using this spectrometer, all peak positions and FWHM values were fixed relative to the Ti $2 \mathrm{p}_{3 / 2}$ peak.

\subsubsection{Niobium}

Quantification of XPS results on niobium described in the literature focuses almost universally on the $\mathrm{Nb} 3 \mathrm{~d}$ peak, which gives a clear pronounced doublet corresponding to the $\mathrm{j}=5 / 2$ and $\mathrm{j}=3 / 2$ spin states [35, 36]. There are three 

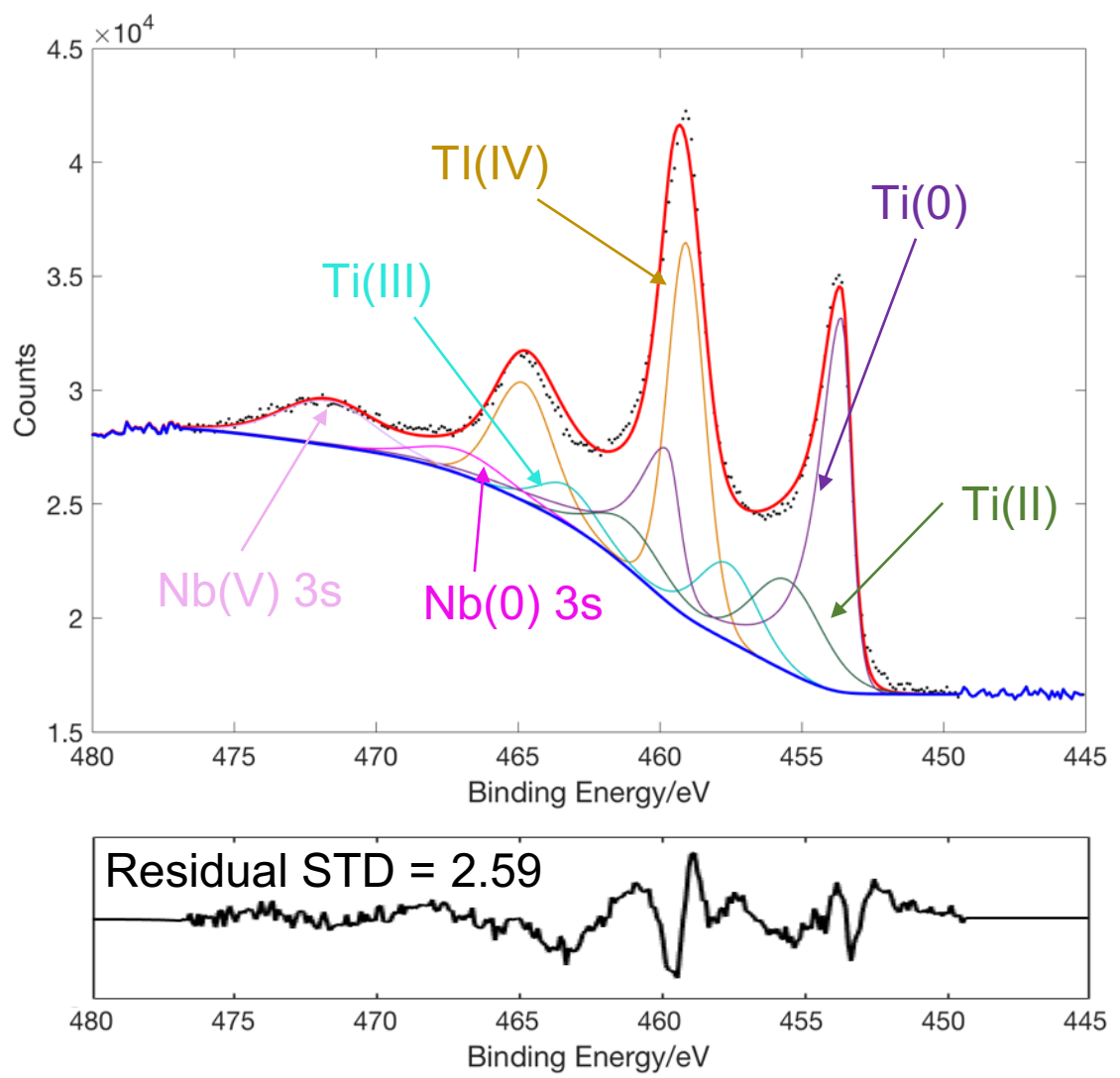

Figure 4: Top: Detailed scan of the titanium 2p region of a NbTi sample oxidised in laboratory air for one minute, taken on the ThermoScientific K-Alpha ${ }^{+}$spectrometer parallel to the surface normal. The contributions to the fit from each of the $\mathrm{Ti}(0), \operatorname{Ti}(\mathrm{II}), \mathrm{Ti}(\mathrm{III})$ and $\mathrm{Ti}(\mathrm{IV})$ oxidation states are shown, along with the extra contributions due to $\mathrm{Nb} 3 \mathrm{~s}$ peaks. Bottom: Plot of the residuals of the fit calculated as the difference between the observed and fitted intensities along with residual standard deviation (STD) values. 
potentially stable oxides commonly expected at low temperatures in the niobium system: $\mathrm{NbO}, \mathrm{NbO}_{2}$ and $\mathrm{Nb}_{2} \mathrm{O}_{5}$. Figure 5 shows the $\mathrm{Nb} 3 \mathrm{~d}$ region from a NbTi sheet in the as-received condition (exposed to air for more than a day, plus exposed to water during the cleaning process) and after sputter etching. The asymmetric metallic doublet clearly visible in the sputter-etched sample has been fitted using an $\operatorname{LA}(1.2,5,12)$ peak shape, after Biesinger [37]. The oxidised peaks are symmetric and have been fitted using a GL(30) peak shape. Small amounts of the metallic peak are still observable in this sample, suggesting any oxide is close to, but not more than, $3 \lambda$ in thickness, though further quantification is not possible owing to the low intensity of the metal signal.
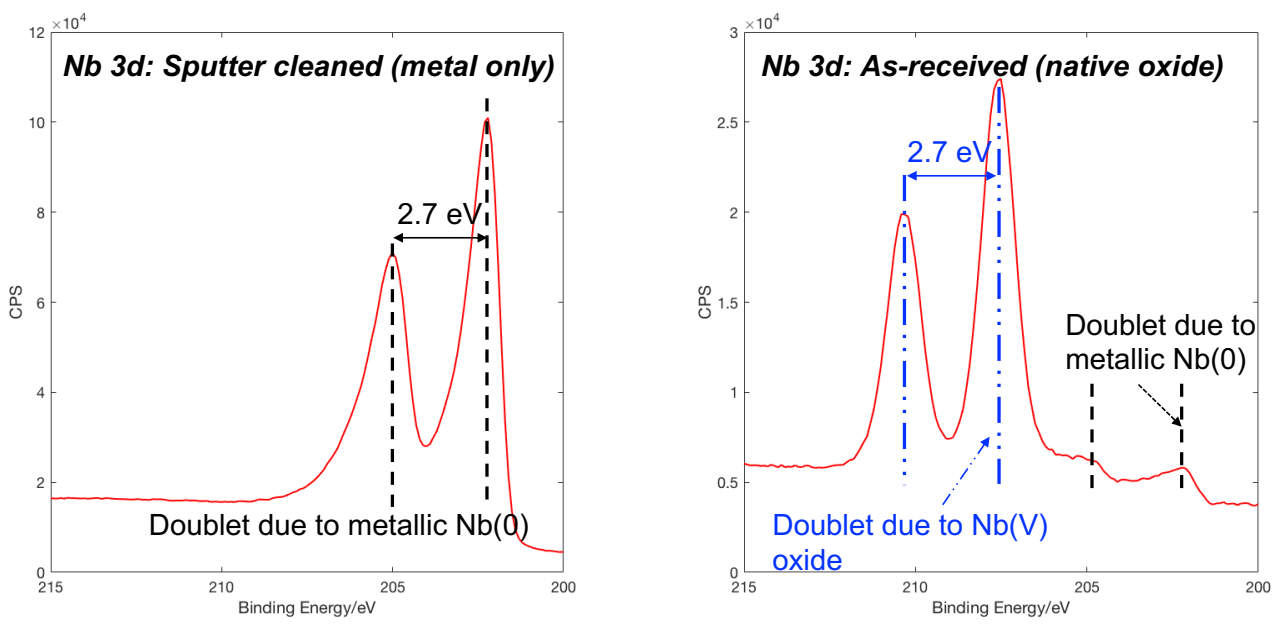

Figure 5: Left: Detailed scan of the niobium 3d region of a sputter cleaned sample taken on the ThermoScientific K-Alpha ${ }^{+}$spectrometer parallel to the surface normal. The metallic doublet demonstrates strong asymmetry. Right: Detailed scan of the niobium 3d region of an as-received sample taken on the Kratos Axis Ultra spectrometer parallel to the surface normal.

Figure 6 shows the detailed fit over the $\mathrm{Nb} 3 \mathrm{~d}$ region for the $\mathrm{NbTi}$ sheet after exposure to air for one minute. There are four clear peaks corresponding to the $\mathrm{Nb}(0)$ and $\mathrm{Nb}(\mathrm{V})$ doublets. However to achieve a good fit, two extra doublets have been added at intermediate binding energies corresponding to 
the $\mathrm{Nb}(\mathrm{II})$ and $\mathrm{Nb}(\mathrm{IV})$ oxidation states. The positions of the $\mathrm{j}=5 / 2$ peaks are listed in Table2 Each of the doublets were successfully fitted with a spin-orbital splitting of $2.72 \mathrm{eV}$, in good agreement with the values described by both Bahl [35] and Fontaine [36. The metal doublet was fitted with the $\operatorname{LA}(1.2,5,12)$ peak shape and the oxide peaks with a GL(30) peak shape. The FWHM of all the oxide peaks was constrained to a single value, whilst the two peaks of the metallic doublet were allowed to differ both from the oxide and from each other, after Olsson 16. However, on fitting it was found that the two peaks in the doublet had approximately the same FWHM, as would be expected. All other oxidation conditions and angles taken using this spectrometer were fitted using this set of fit parameters, keeping the same relative energy separations between peaks and the same relative FWHM values. The position of the entire fit was 210 allowed to vary between samples to allow for any minor charging effects. 


\begin{tabular}{ccccc}
\hline Species & Binding Energy/eV & Peak Splitting/eV & FWHM $/ \mathrm{eV}$ & Peak Shape \\
\hline $\operatorname{Ti}(0) 2 \mathrm{p}$ & 453.59 & 6.15 & $0.85(1.10)$ & $\mathrm{LA}(1.1,5,7)$ \\
$\operatorname{Ti}(\mathrm{II}) 2 \mathrm{p}$ & +1.91 & 5.60 & 2.95 & $\mathrm{GL}(30)$ \\
$\mathrm{Ti}(\mathrm{III}) 2 \mathrm{p}$ & +4.00 & 5.62 & 2.47 & $\mathrm{GL}(30)$ \\
$\mathrm{Ti}(\mathrm{IV}) 2 \mathrm{p}$ & +5.48 & 5.72 & $1.55(2.45)$ & $\mathrm{GL}(30)$ \\
$\mathrm{Nb}(0) 3 \mathrm{~s}$ & +13.39 & $\mathrm{n} / \mathrm{a}$ & 3.72 & $\mathrm{GL}(30)$ \\
$\mathrm{Nb}(\mathrm{V}) 3 \mathrm{~s}$ & +18.12 & $\mathrm{n} / \mathrm{a}$ & 3.91 & $\mathrm{GL}(30)$ \\
\hline
\end{tabular}

Table 1: Summary of the fit parameters used for the titanium $2 \mathrm{p}$ region of a NbTi sample oxidised in laboratory air for one minute and measured in the ThermoScientific K-Alpha ${ }^{+}$ spectrometer parallel to the surface normal. For some fits the FWHM of the doublet peaks was allowed to differ. For these the FWHM of the $1 / 2$ spin peak is shown in brackets. The same fit was transferred to all other samples in this series, with only the total FWHM and position (but not the relative spacing) allowed to vary.

\begin{tabular}{ccccc}
\hline Species & Binding Energy/eV & Peak Splitting/eV & FWHM/eV & Peak Shape \\
\hline $\mathrm{Nb}(0) 3 \mathrm{~d}_{5 / 2}$ & 202.1 & 2.72 & 0.77 & $\mathrm{LA}(1.2,5,12)$ \\
$\mathrm{Nb}(\mathrm{II}) 3 \mathrm{~d}_{5 / 2}$ & +1.88 & 2.72 & 1.50 & GL $(30)$ \\
$\mathrm{Nb}(\mathrm{IV}) 3 \mathrm{~d}_{5 / 2}$ & +3.77 & 2.72 & 1.50 & $\mathrm{GL}(30)$ \\
$\mathrm{Nb}(\mathrm{V}) 3 \mathrm{~d}_{5 / 2}$ & +5.41 & 2.72 & 1.50 & $\mathrm{GL}(30)$ \\
\hline
\end{tabular}

Table 2: Summary of the fit used for the niobium 3d region of a NbTi sample oxidised in laboratory air for one minute. The same fit was transferred to all other samples in this series, with only the total FWHM and position (but not the relative spacing) allowed to vary. 

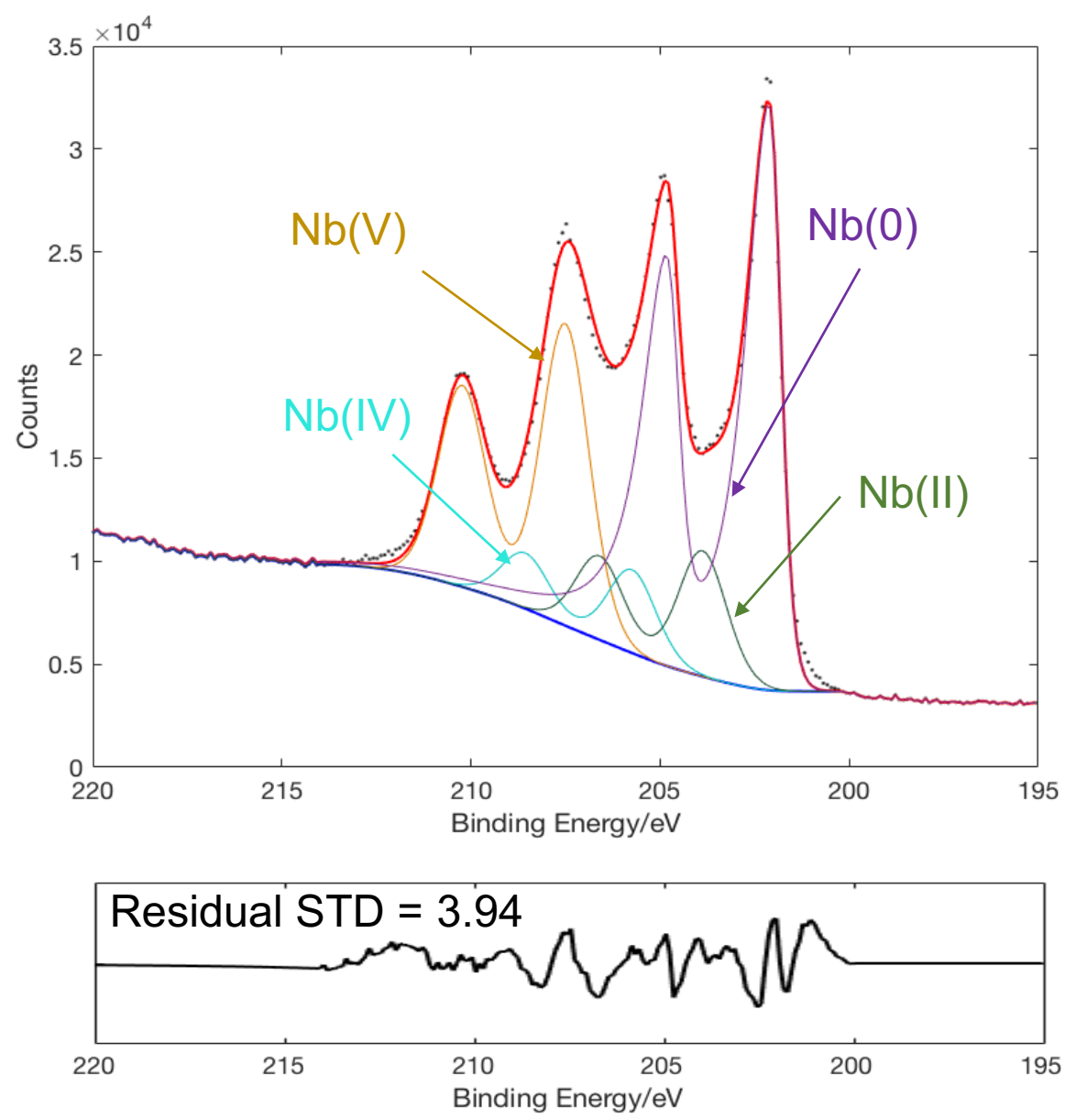

Figure 6: Top: Detailed scan of the niobium 3d region of a NbTi sample oxidised in laboratory air for one minute taken on the ThermoScientific K-Alpha ${ }^{+}$spectrometer parallel to the surface normal. The contributions to the fit from each of the $\mathrm{Nb}(0), \mathrm{Nb}(\mathrm{II}), \mathrm{Nb}(\mathrm{IV})$ and $\mathrm{Nb}(\mathrm{V})$ oxidation states are shown. Bottom: Residual plot from the fit along with residual standard deviation (STD) values. 


\subsection{Air Oxidation Experiments}

Figure 7 shows the detailed normal-emission spectra for oxidation times between 15 and 3600 seconds. After the shortest exposure time there are already two distinct doublets present for both niobium and titanium corresponding to the formation of oxide on the surface of the NbTi sheet, even with the spectrometer at this most bulk-sensitive angle. As oxidation time increases, the intensity of the higher binding energy oxide peaks increases at the expense of the lower binding energy metallic doublet. This is most obvious in the Nb spectra due to the absence of peak overlaps, but it is also observable in the Ti spectra.
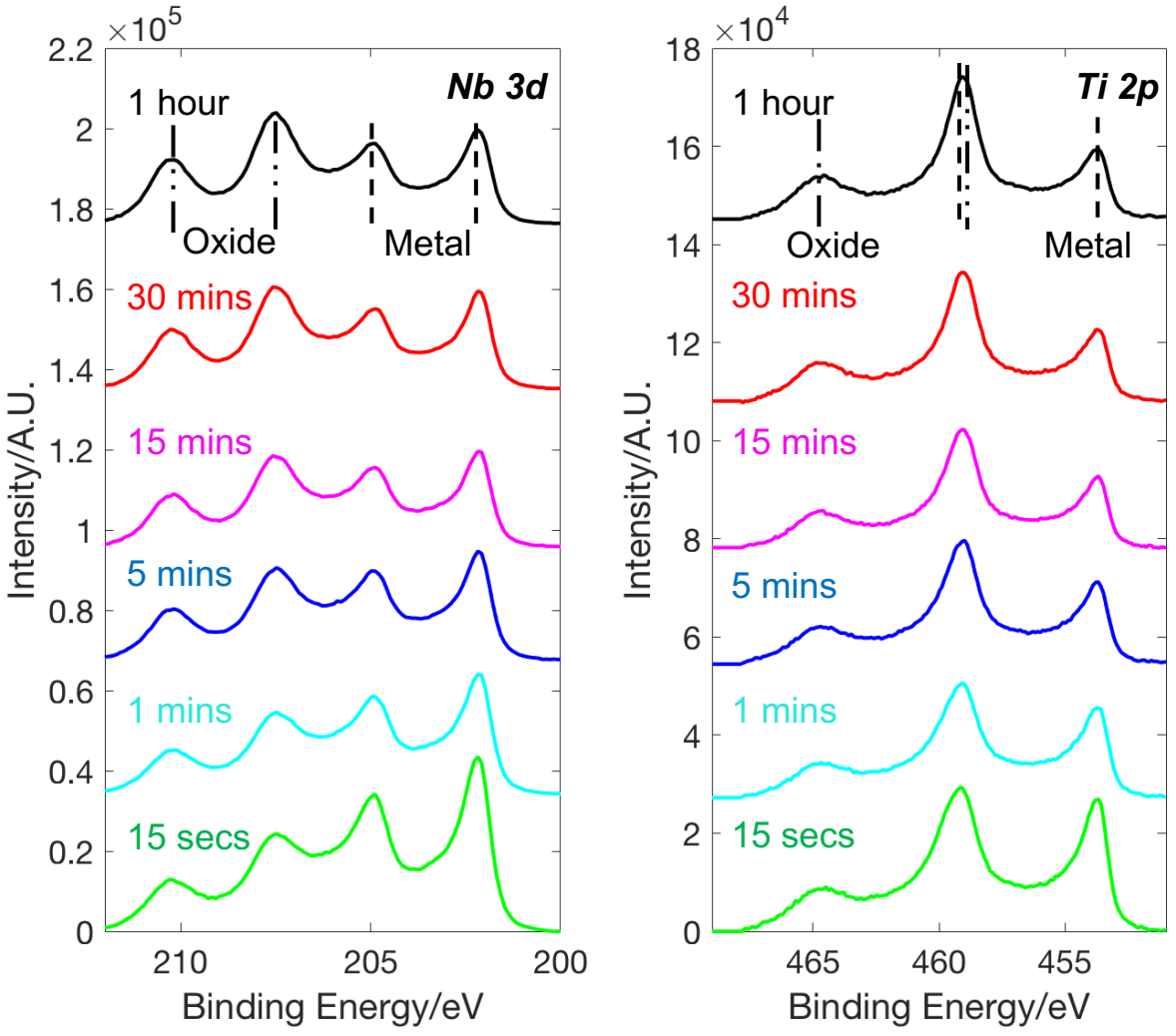

Figure 7: Detailed scans of the niobium 3d and titanium 2p regions of a NbTi sample oxidised in laboratory air for times between 15 seconds and 1 hour taken on the ThermoScientific $\mathrm{K}-\mathrm{Alpha}^{+}$spectrometer at normal emission angle 
The spectra taken at increasing emission angles from $0^{\circ}$ to $70^{\circ}$ are shown in Figure 8 for the sample oxidised for 1 minute. The more bulk-sensitive low emission angle measurements demonstrate a more intense metal peak compared to the oxide peak, whilst the opposite is true at high emission angles. This indicates that the oxide is located at the surface of the sample, as expected. Similar trends appear in both the niobium and the titanium data, confirming the presence of a mixed $\mathrm{Nb} / \mathrm{Ti}$ oxide on the surface, rather than an oxide dominated by one species as is observed in some metallic alloys such as stainless steels [38.

$\mathrm{Nb} 3 d$

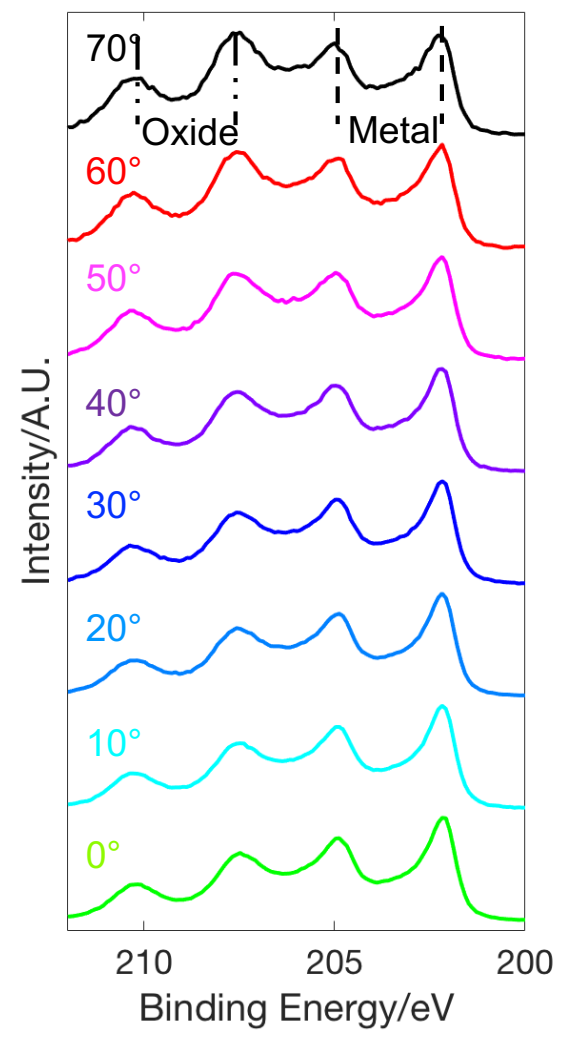

Ti $2 p$

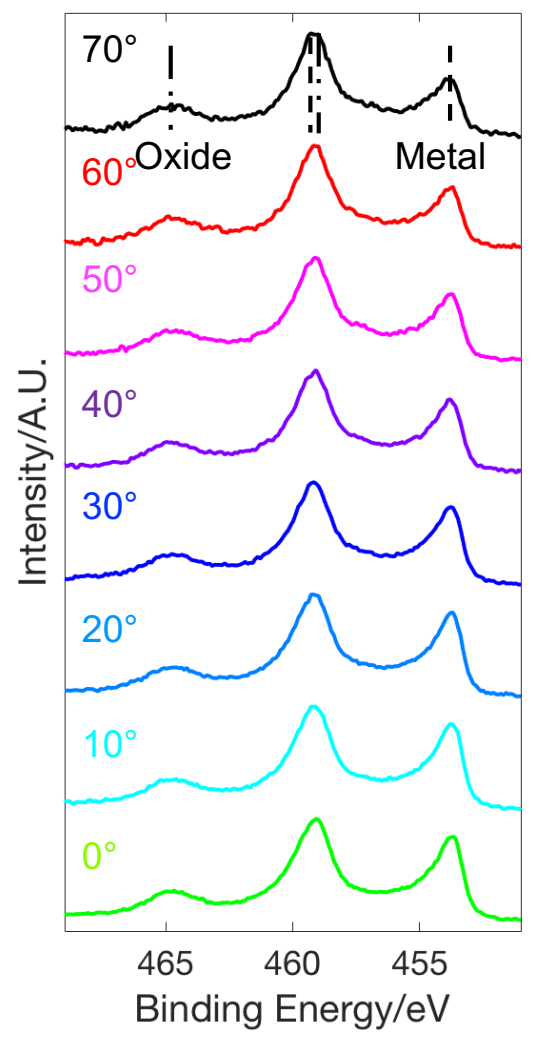

Figure 8: Detailed scans of the niobium 3d and titanium 2p regions of a NbTi sample oxidised in laboratory air for 1 minute taken on the ThermoScientific $\mathrm{K}^{-A_{l}} \mathrm{pha}^{+}{ }^{+}$with detection angles between $0^{\circ}$ and $70^{\circ}$ from the surface normal 


\subsubsection{Oxide Composition and Structure}

Understanding the chemical composition of the oxide that forms on the NbTi is crucial to understanding how the presence of a thin layer may affect the superconducting properties of a persistent mode joint. Here the sample oxidised for 1 minute is analysed in detail, and these results are similar to that obtained from other samples with different oxide thicknesses.

Figure $9 \mathrm{a}$ shows the relative composition of the three main expected components of the surface after exposure to air for 1 minute extracted using equation 2 There is also approximately $10 \%$ carbon present in each sample, mostly due to the presence of an organic contamination layer from the exposure to air, which has not been included in the composition analysis. The concentration of oxygen increases slightly with increasing emission angle at the expense of the metal species, indicating a higher concentration of oxygen at the surface. However, since all of the niobium and titanium oxides will contribute to the oxygen signal, more detailed understanding of the nature of the oxide layer/s can only be obtained by examining the $\mathrm{Nb}$ and Ti spectra. Figure $9 \mathrm{~b}$ shows the $\mathrm{Nb}$ and $\mathrm{Ti}$ concentrations as a function of angle on an expanded scale. There is no evidence of a significant change in the relative concentrations of $\mathrm{Nb}$ or $\mathrm{Ti}$ with emission angle, suggesting the ratio of $\mathrm{Nb}: \mathrm{Ti}$ in the oxide is similar to that in the base alloy. The concentration of titanium in the base metal and oxide appears lower than expected, consistently around 45 at\% in all samples measured in the XPS. This contrasts with 61 at\% measured by EDX. This is attributed to systematic errors introduced by the assumption that the material is homogeneous, the use of sensitivity factors derived from results on pure elements rather than alloys, and errors introduced by the background subtraction.

Detailed fitting of the $\mathrm{Nb}$ and $\mathrm{Ti}$ spectra using the procedure given in section 4.1 has been performed to gain a greater understanding of the composition of the oxide layer. Figure 10 plots the ratios of the measured peak areas of each of the peaks attributable to the $\mathrm{Ti}$ and $\mathrm{Nb}$ in each oxidation state relative to the metallic (0) state. There is a sharp increase with analysis angle in the intensity 

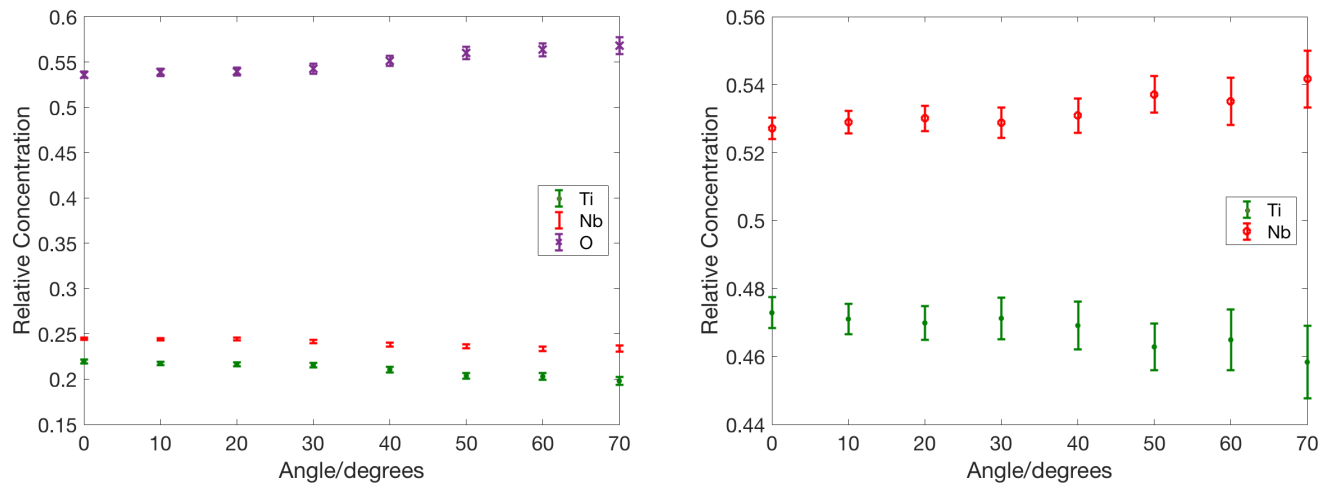

Figure 9: Relative concentrations calculated using the total intensity of a) $\mathrm{O} 1 \mathrm{~s}, \mathrm{Nb} 3 \mathrm{~d}$ and Ti 2p peaks and b) just the Nb 3d and Ti 2p peaks for the NbTi sample oxidised in laboratory air for 1 minute. This data was taken on the ThermoScientific K-Alpha ${ }^{+}$with detection angles between 0 and $70^{\circ}$ from the surface normal

of the peaks characteristic of the highest oxidation state for both niobium and titanium, indicating that these species are located preferentially at the surface of the material. A weaker increasing trend is also discernible for the intermediate oxidation states components, $\mathrm{Nb}(\mathrm{II}), \mathrm{Nb}(\mathrm{IV}), \mathrm{Ti}(\mathrm{II})$ and $\mathrm{Ti}$ (III), suggesting that these lower oxides are physically located between the higher oxides and the base metal.

\subsubsection{Thickness calculations}

While Figure 10 suggests that the lower oxides are located below the higher oxides, their intensities are low meaning they are relatively thin. This introduces large errors in the peak fitting, particularly for the titanium species, making it difficult to quantify the relative depth of the different species. Therefore a reasonable approximation seems to be to treat the oxide as a single layer with the properties of the outer oxidation states, but combining the intensities of all the oxide peaks to estimate the total thickness of all the oxide layers. The mixed nature of the oxide containing both $\mathrm{Nb}$ and $\mathrm{Ti}$ also has the potential to complicate the picture. In the Strohmeier equation (equation 5), this can be accounted for by adjusting the values of the atomic density of the metal and 

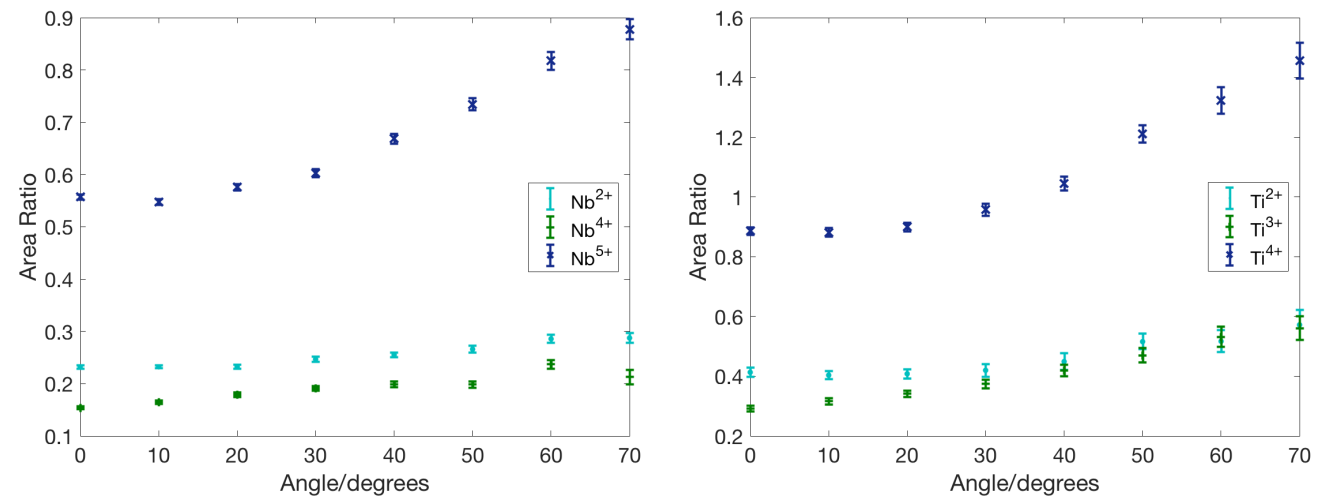

Figure 10: Relative area ratios $\left(\mathrm{A}_{O x} / \mathrm{A}_{\text {Met }}\right)$ for a) niobium and b) titanium oxide species for the NbTi sample oxidised in laboratory air for 1 minute taken on the ThermoScientific $\mathrm{K}-\mathrm{Alpha}^{+}$with detection angles between $0^{\circ}$ and $70^{\circ}$ from the surface normal.

oxide $N_{m e t}$ and $N_{o x}$ by the atomic fraction of $\mathrm{Nb}$ and $\mathrm{Ti}$ in each oxide such that the ratio becomes $f_{m e t} N_{m e t} / N_{o x} f_{o x}$. In Figure 9 it can be seen that $f_{m e t}$ and $f_{o x}$ are approximately equal, so these factors cancel out. Assuming the oxide is mixed in this way, the thickness has been calculated independently from both the niobium and titanium data.

The values of the parameters used for the calculation are shown in Table 3. Values for the inelastic mean free path were calculated using the TPP-2M formulation [39] for each species in both the metal and oxide. The atomic densities were approximated from bulk lattice parameters, noting that this may differ from the atomic densities in thin amorphous oxide layers.

\begin{tabular}{ccccc}
\hline Element & $\lambda_{o x} / \mathrm{nm}$ & $\lambda_{\text {met }} / \mathrm{nm}$ & $N_{o x} / \mathrm{mol} \mathrm{cm}^{-3}$ & $N_{\text {met }} / \mathrm{mol} \mathrm{cm}^{-3}$ \\
\hline $\mathrm{Nb}$ & 3.3 & 2.8 & 0.035 & 0.092 \\
$\mathrm{Ti}$ & 2.7 & 2.3 & 0.053 & 0.094 \\
\hline
\end{tabular}

Table 3: Summary of the parameters used in the Strohmeier equation for niobium and titanium in the calculation of the oxide thickness

Theoretically the Strohmeier equation predicts a linear relationship between $1 / \cos \theta$ and $\ln \left[1+\frac{\lambda_{m e t} N_{m e t}}{\lambda_{o x} N_{o x}} \frac{I_{o x}}{I_{m e t}}\right]$ with gradient $t_{o x} / \lambda_{o x}$. However, Figure 11 
shows that neither the titanium nor the niobium data fit this theoretical model, with the observed deviation from linearity corresponding to a decrease in calculated thicknesses at high emission angles. This has been observed previously for pure $\mathrm{Nb}$ by Darlinski [40. Gunter has attributed this observation to surface roughness, and through a combination of modelling and experiment demonstrated that these effects could be minimised by using an emission angle close to the "magic" angle of $40^{\circ}$ [41, 42. Normalising our calculated oxide thicknesses against the corresponding data at $40^{\circ}$ all of the measurements are found to collapse onto a single curve (see supplementary information [27]), suggesting that the observed deviations can be explained by surface roughness effects. Therefore, in the following analysis thickness values have been calculated using spectra taken at Gunter's "magic angle" of $40^{\circ}$.

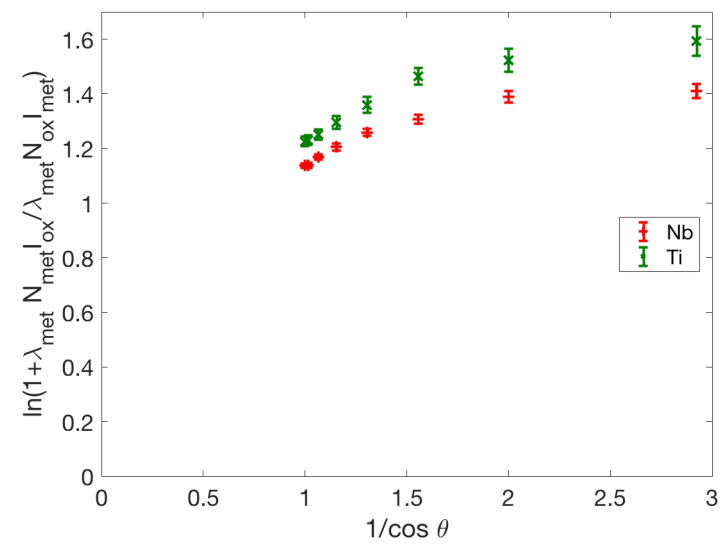

Figure 11: Strohmeier plot for the intensity ratios calculated as the sum of all oxide contributions for the niobium and titanium components.

The thicknesses calculated separately from the niobium and titanium data at $40^{\circ}$ from the surface normal are shown in Figure $12 \mathrm{a}$. The separate calculations for niobium and titanium give similar results, which suggests the initial assumptions about the nature of the oxide layer are reasonable. In the first 15 seconds of oxidation, a $2.5 \mathrm{~nm}$ oxide forms, which thickens to $3.5 \mathrm{~nm}$ after an hour (based on the Ti data). There is a consistent overestimate of approxi- 
mately $10 \%$ from the niobium data compared to the titanium data. This may be due to slight compositional variations in the oxide compared to the bulk (if the oxide is slightly niobium enriched). However, any systematic error in the estimation of the inelastic mean-free path, often quoted to be accurate to only about $\pm 10 \%$ for elements and nearly $\pm 20 \%$ for inorganic compounds such as oxides [39], would affect all the data for each element separately but not affect the trend. This uncertainty in the absolute values is sufficient to account for the difference in thickness calculated from the niobium and titanium data.

The oxide thickness, $d$, as a function of time, $t$, fits well to a logarithmic model $d=a \ln t+c$, as shown in Figure $12 \mathrm{~b}$. This is in good agreement with data reported by Grundner [15] for pure niobium samples, which demonstrates similar growth kinetics for short oxidation times. Grundner correlates this to a growth mechanism limited by chemisorption or oxygen diffusion through the oxide. This mechanism is described in detail by Boggio for thin aluminium films [43. Cabrera and Mott 44] suggest an alternative growth model for oxidation of 20 reactive metals at low temperatures, $1 / d=c-a \ln t$, which takes into account the effect of electric field in the oxide layer on the movement of metal ions. However, Figure 12 c shows that this model is a poorer fit to our data.

Our calculated oxide thicknesses are generally higher than those reported by Grundner, who reports $\mathrm{Nb}_{2} \mathrm{O}_{5}$ thicknesses of approximately 1-1.5 nm after 10 325 minutes, depending on sample preparation [15]. Although direct comparisons are difficult as full consideration of the lower oxides has not been reported for all samples, the differences can be attributed to our samples being oxidised in wet laboratory air rather than dry air, which is known to lead to faster oxidation in both niobium [15] and titanium [45]. In addition, the sputter etching of our samples is likely to have introduced a high surface defect density, which may have led to rapid initial oxidation [15]. 
a)

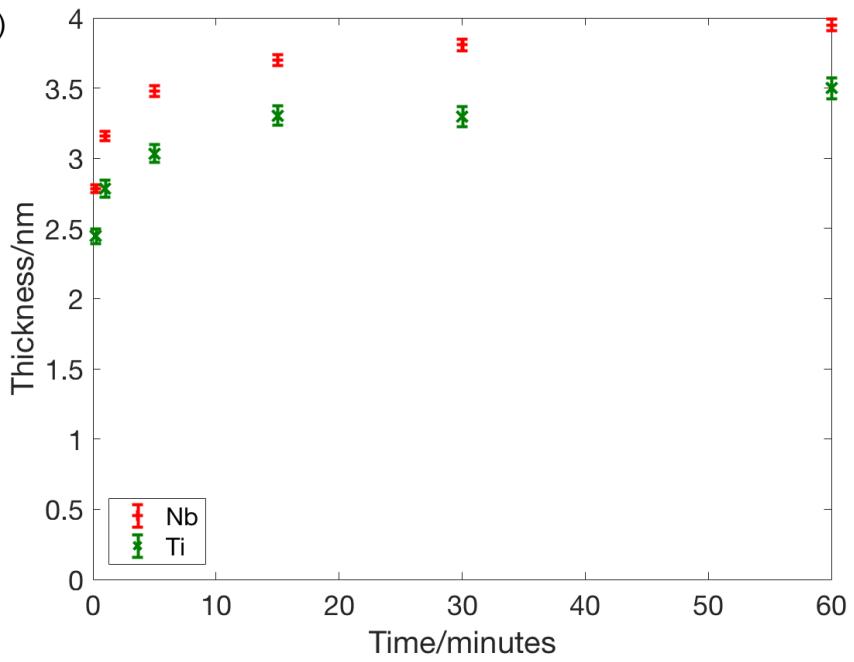

b)

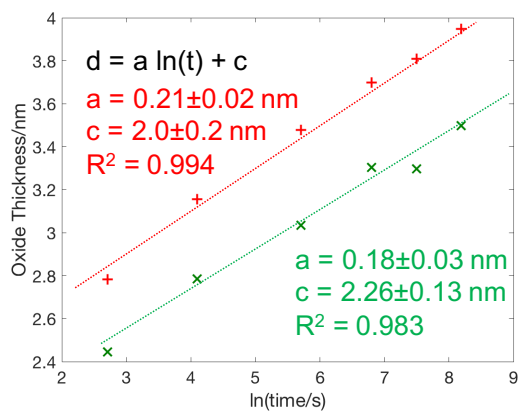

c)

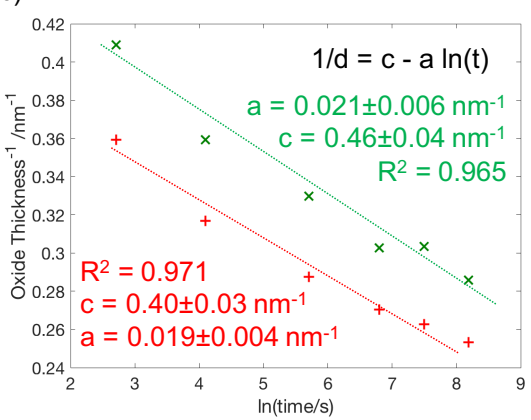

Figure 12: a) Oxide thickness calculated using the Strohmeier equation (Equation 5 for a NbTi sheet oxidised in air for different times. All measurements were calculated from data taken with the angle of $40^{\circ}$ between the detector and sample surface normal. The error bars apply only to random errors resulting from the fitting of the curves. b) The same data set plotting logarithmic data $\ln (t)$ against thickness and fitted according to Boggio's model [43]. c) The same data set plotting $\ln (t)$ against $1 /$ thickness and fitted according to the Cabrera-Mott model [4] 


\subsection{Gas Dosing Experiments}

To gain a better understanding of the earliest stages of oxidation, controlled gas dosing experiments were performed as described in Section 3.2 . To accua later stage of oxidation, more care must be taken when analysing the gas- 
a)

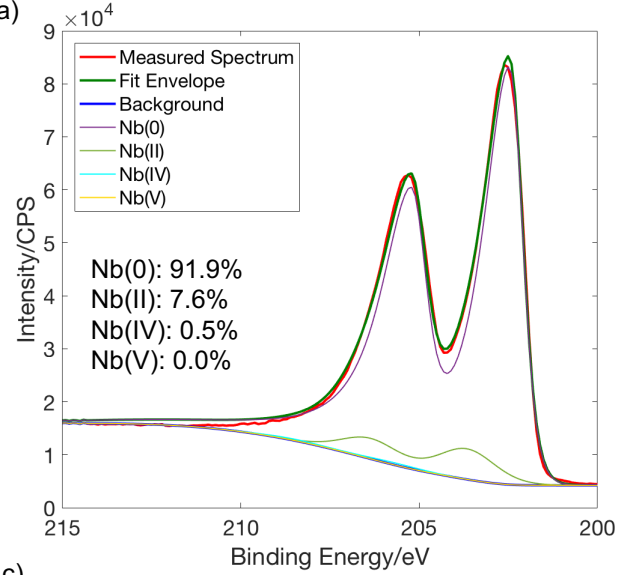

c)

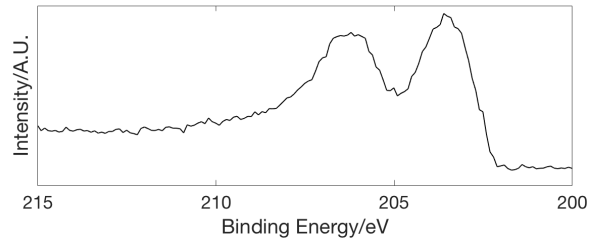

b)

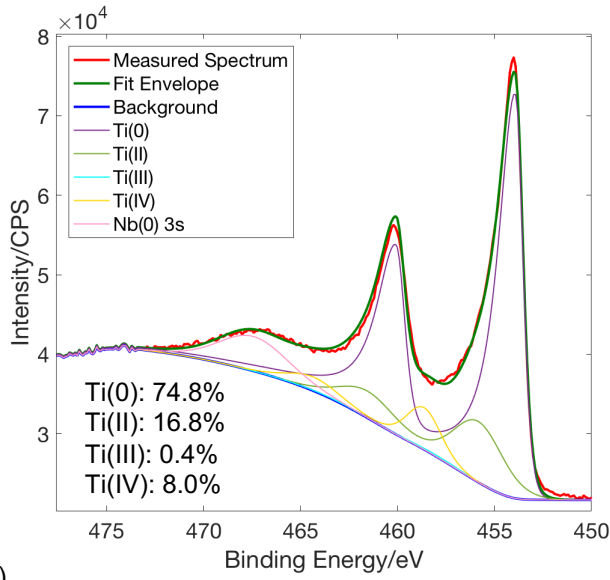

d)

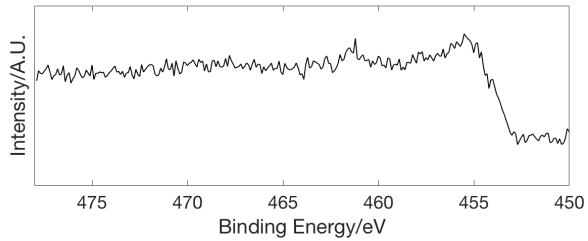

Figure 13: Detailed spectra of a) the $\mathrm{Nb} 3 \mathrm{~d}$ and b) the Ti 2p region from a sputter cleaned NbTi sheet measured parallel to the surface normal in the Kratos Axis Ultra spectrometer. The curve fits used for these are shown, demonstrating the presence of smallconcentrations of oxidised species. The fitted area fractions fitted of all the species shown (note that the contribution from the $\mathrm{Nb} 3 \mathrm{~s}$ peak is ignored for the titanium sample). c) and d) are difference spectra taken by subtracting the spectra measured at emission angle of $60^{\circ}$ from the spectra taken at normal emission for the niobium and titanium regions respectively

dosing experiments where the presence of this initial surface oxide must be taken into account.

The results from all the experiments at the most surface-sensitive angle, $60^{\circ}$ from the surface normal, are shown in Figure 14. For the low gas doses, $<1000 \mathrm{~L}$, there is little change in which peaks are present, with only a slight broadening of the metallic peak. This indicates the growth of lower oxides, such as $\mathrm{Nb}(\mathrm{II})$ and $\mathrm{Ti}(\mathrm{II})$, which overlap with the metallic peaks. At $10000 \mathrm{~L}$ there a clear emergence of new peaks, marked by arrows in Figure 14, corresponding to the presence of significant quantities of higher $\mathrm{Ti}(\mathrm{IV})$ and $\mathrm{Nb}(\mathrm{V})$ oxides.

Relative peak areas are shown in Figure 15 for the oxidised species nor- 
malised by the intensity of the equivalent metallic peak for measurements taken at $60^{\circ}$ emission angle. The low oxygen exposures lead to a clear increase in 375 the $\mathrm{Nb}(\mathrm{II})$ and $\mathrm{Ti}(\mathrm{II})$ oxidation states corresponding to the growth of a mixed $\mathrm{NbO}$ and $\mathrm{TiO}$ layer. There is also a gradual increase in the $\mathrm{Nb}(\mathrm{V})$ and $\mathrm{Ti}(\mathrm{IV})$ intensities up until $10000 \mathrm{~L}$, followed by an abrupt increase corresponding to the emergence of the new peaks in Figure 14 as the outer layer of the higher oxide forms. This is in reasonable agreement with Carley's results for gas-dosed pure titanium 47, in which a fast initial growth of $\mathrm{Ti}(\mathrm{II})$ oxide was found to stabilise after a small dose, followed by a more gradual thickening of Ti(IV) oxide, with similar concentrations of outer and sub-oxide species observed after a dose of $12000 \mathrm{~L}$.

Due to uncertainties in the exact energies and lineshapes of the different components used to fit the samples, care must be taken when comparing niobium and titanium results. However, it appears that the intensity ratio of $\mathrm{Nb}$ (II) increases more slowly than $\mathrm{Ti}(\mathrm{II})$ with oxygen dose, suggesting that the initial oxide formed may be more Ti-rich, with the $\mathrm{Nb}$ (II) components only forming at higher oxygen doses. 

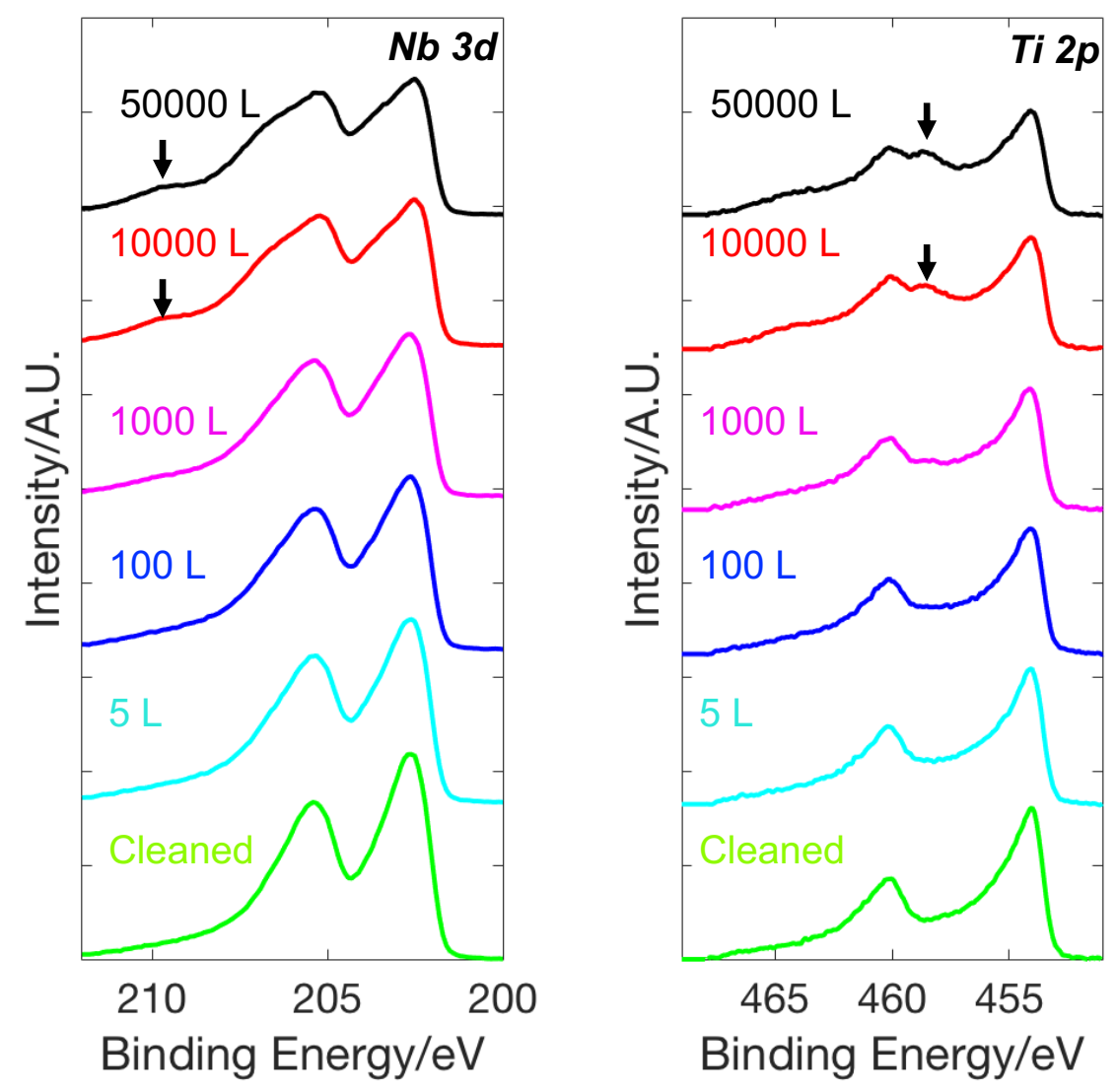

Figure 14: Detailed spectra of the Nb 3d and Ti 2p regions from a NbTi sheet measured at $60^{\circ}$ to the surface normal in the Kratos Axis Ultra spectrometer as cleaned and after exposure to between 5 and $50000 \mathrm{~L}$. The emergence of distinct $\mathrm{Ti}(\mathrm{IV})$ and $\mathrm{Nb}(\mathrm{V})$ peaks, highlighted with arrows, becomes apparent after about $10000 \mathrm{~L}$. 
a)

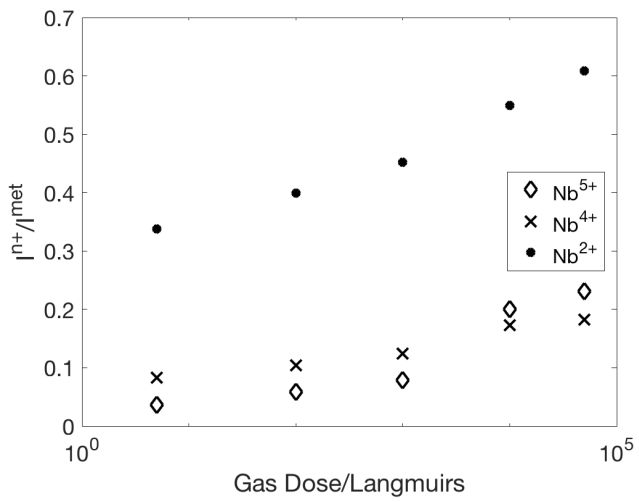

b)

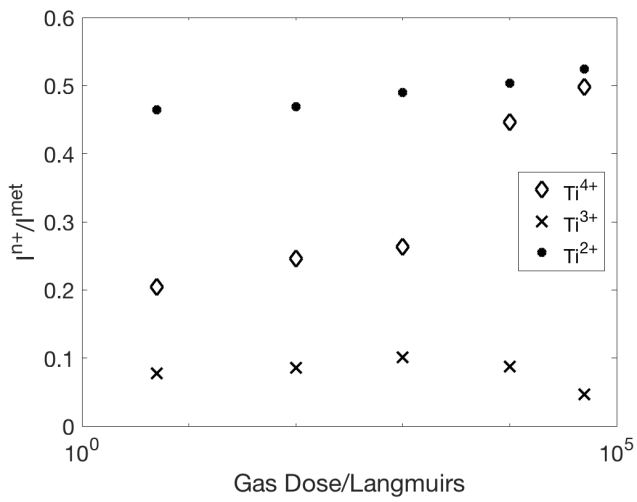

Figure 15: Intensity ratios of oxidised a) niobium and b) titanium species normalised by the intensity of the metal peaks after different gas doses calculated from the $\mathrm{Nb} 3 \mathrm{~d}$ and $\mathrm{Ti} 2 \mathrm{p}$ spectral regions from a NbTi sheet measured at an emission angle of $60^{\circ}$ in the Kratos Axis Ultra spectrometer after exposure to oxygen doses between 5 and $50000 \mathrm{~L}$ 


\section{Conclusions}

Detailed XPS studies into the oxidation of $\mathrm{Nb}-45 \mathrm{wt} \% \mathrm{Ti}$ have been performed using a combination of ambient air exposure and gas dosing experiments. Samples exposed to laboratory air for times between 15 seconds and 1 hour demonstrate significant oxidation, forming layers approximately 2.5-2.8 nm thick after 15 seconds and thickening following $d \propto \ln t$ to a limiting thickness of $\approx 3.5 \mathrm{~nm}$. This is of concern for industrial production of NbTi joints, where exposure of the filaments to air for these timescales may be difficult to avoid. The superconducting coherence length in NbTi is $\approx 5 \mathrm{~nm}$, and so oxide layers a few nm thick will form a non-superconducting barrier to current flow, and are expected to introduce significant resistance into a joint. The ratio of $\mathrm{Nb}$ :Ti of the oxide was found to be similar to that of the bulk alloy, suggesting no preferential oxidation of either $\mathrm{Nb}$ or $\mathrm{Ti}$. This detailed chemical analysis may help guide future modelling of the effect of the presence the oxide layer on the superconducting properties of joints.

Experiments using low doses of oxygen demonstrated the formation of $\mathrm{Nb}(\mathrm{V})$ and $\mathrm{Ti}(\mathrm{IV})$ layers after doses of $10000 \mathrm{~L}$, equivalent to approximately $50 \mu \mathrm{s}$ in air. This demonstrates that keeping a NbTi surface oxide-free is practically impossible. Some signs of oxide were found even on Ar-beam cleaned samples, demonstrating that storage of NbTi under anything other than a highly reducing

\section{Acknowledgement}

We gratefully acknowledge provision by the EPSRC of the X-ray photoelectron Spectroscopy (XPS) service hosted by Cardiff University through the "Access to Research Equipment Initiative" (EP/F019823/1) and would like to 
thank Diamond Light Source for beamtime (proposal SI18882-1) and the staff

of beamline I09 for assistance with data collection.

\section{References}

[1] Z. Melhem, Materials uk preliminary review superconducting materials and applications a uk challenge and an opportunity (2011).

[2] R. M. Scanlan, A. P. Malozemoff, D. C. Larbalestier, Superconducting materials for large scale applications, Proc. IEEE 92 (10) (2004) 1639-1654 (2004).

[3] C. Swenson, W. Markiewicz, Persistent joint development for high field NMR, IEEE Trans. Appiled Supercond. 9 (2) (1999) 185-188 (1999).

[4] R. F. Thornton, Superconducting joint for superconducting wires and coils and method of of forming, patent US4907338 (1990).

[5] M. Kodama, K. Okamoto, Y. Koga, T. Yamamoto, H. Watanabe, Analysis for formation of current path in the superconducting joint between $\mathrm{Nb}-\mathrm{Ti}$ wires with the solder matrix replacement method, Supercond. Sci. Technol. 28 (4) (2015) 045019 (2015).

${ }_{435}$ [6] L174/88, Off. J. Eur. Union (2011).

[7] L4/61, Off. J. Eur. Union (2014).

[8] M. J. Leupold, Y. Iwasa, Superconducting joint between multifilamentary wires 1. Joint-making and results, Cryogenics (Guildf). 16 (4) (1976) 215216 (1976).

[9] J. Liu, J. Cheng, F. Zhou, Q. Wang, K. Chang, X. Li, Electrical properties of cold-pressing welded NbTi persistent joints, Cryogenics (Guildf). 58 (2013) 62-67 (2013). 
[10] K. Seo, S. Nishijima, K. Katagiri, T. Okada, Evaluation of solders for superconducting magnetic shield, IEEE Trans. Magn. 27 (2) (1991) 18771880 (1991).

[11] P. J. Cumpson, Angle-resolved XPS and AES: Depth-resolution limits and a general comparison of properties of depth-profile reconstruction methods, J. Electron Spectros. Relat. Phenomena 73 (1) (1995) 25-52 (1995).

[12] L. P. H. Jeurgens, M. S. Vinodh, E. J. Mittemeijer, Initial oxide-film growth on Mg-based MgAl alloys at room temperature, Acta Mater. 56 (17) (2008) 4621-4634 (2008).

[13] E. Panda, L. P. H. Jeurgens, E. J. Mittemeijer, Growth kinetics and mechanism of the initial oxidation of Al-based Al-Mg alloys, Corros. Sci. 52 (8) (2010) 2556-2564 (2010).

[14] S. Suzuki, K. Yanagihara, K. Hirokawa, XPS study of oxides formed on the surface of high-purity iron exposed to air, Surf. Interface Anal. 30 (1) (2000) 372-376 (2000).

[15] M. Grundner, J. Halbritter, On the natural Nb2O5 growth on Nb at room temperature, Surf. Sci. 136 (1984) 144-154 (1984).

[16] C. O. A. Olsson, D. Landolt, Atmospheric oxidation of a Nb-Zr alloy studied with XPS, Corros. Sci. 46 (1) (2004) 213-224 (2004).

[17] J. Halbritter, Low temperature oxidation of $\mathrm{Nb}$ and of Nb-compounds in relation to superconducting application, J. Less-Common Met. 139 (1) (1988) 133-148 (1988).

[18] Q. Ma, R. A. Rosenberg, Angle-resolved X-ray photoelectron spectroscopy study of the oxides on $\mathrm{Nb}$ surfaces for superconducting r.f. cavity applications, Appl. Surf. Sci. 206 (1-4) (2003) 209-217 (2003).

[19] E. McCafferty, J. Wightman, An X-ray photoelectron spectroscopy sputter profile study of the native air-formed oxide film on titanium, Appl. Surf. Sci. 143 (1-4) (1999) 92-100 (1999). 
[20] M. F. Sunding, I. T. Jensen, P. M. Stenstad, S. Diplas, ARXPS and high energy XPS characterisation of titanium surfaces for medical implants, Surf. Interface Anal. 40 (3-4) (2008) 751-753 (2008).

[21] A. Einstein, Über einen die erzeugung und verwandlung des lichtes betreffenden heuristischen gesichtspunkt, Annalen der Physik 17 (6) (1905) 132-148 (1905).

[22] J. H. Scofield, Hartree-Slater subshell photoionization cross-sections at 1254 and 1487 eV, J. Electron Spectros. Relat. Phenomena 8 (2) (1976) 129-137 (1976).

[23] D. F. Mitchell, K. B. Clark, J. A. Bardwell, W. N. Lennard, G. R. Massoumi, I. V. Mitchell, Film Thickness Measurements of SiO2 by XPS, Surf. Interface Anal. 21 (August 1993) (1994) 44-50 (1994).

[24] B. R. Strohmeier, an Esca Method for Determining the Oxide Thickness on Aluminum-Alloys, Surf. Interface Anal. 15 (1) (1990) 51-56 (1990).

[25] C. Chen, S. J. Splinter, T. Do, N. S. McIntyre, Measurement of oxide film growth on $\mathrm{Mg}$ and $\mathrm{Al}$ surfaces over extended periods using XPS, Surf. Sci. 382 (1-3) (1997) L652-L657 (1997).

[26] P. J. Lee, Abridged Metallurgy of Ductile Alloy Superconductors (1999).

[27] Supplementary material [online].

[28] N. Fairley, CasaXPS Manual 2.3.15, Casa Software Limited.

[29] S. Tougaard, Quantitative analysis of the inelastic background in surface electron spectroscopy, Surf. Interface Anal. 11 (9) (1988) 453-472 (1988).

[30] P. van der Heide, X-ray Photoelectron Spectroscopy: An Introduction to Principles and Practices, Wiley, 2012 (2012).

[31] P. Kofstad, High-temperature oxidation of titanium, J. Less Common Met. 12 (6) (1967) 449-464 (1967). 
[32] N. R. Armstrong, R. K. Quinn, Auger and X-ray photoelectron spectroscopic and electrochemical characterization of titanium thin film electrodes, Surf. Sci. 67 (2) (1977) 451-468 (1977).

[39] S. Tanuma, C. J. Powell, D. R. Penn, Calculations of electron inelastic mean free paths. V. Data for 14 organic compounds over the $50-2000 \mathrm{eV}$ range, Surf. Interface Anal. 21 (3) (1994) 165-176 (1994).

[40] A. Darlinski, J. Halbritter, Angle-resolved XPS studies of oxides at NbN ,

[41] P. L. J. Gunter, J. W. Niemantsverdriet, Thickness determination of uniform overlayers on rough substrates by angle-dependent XPS, Appl. Surf. Sci. 89 (1) (1995) 69-76 (1995). 
[42] P. Gunter, O. Gijzeman, J. Niemantsverdriet, Surface roughness effects in quantitative XPS: magic angle for determining overlayer thickness, Appl. Surf. Sci. 115 (4) (1997) 342-346 (1997).

[43] J. E. Boggio, R. C. Plumb, Theory of formation of very thin oxide films on metals, The Journal of Chemical Physics 44 (3) (1966) 1081-1086 (1966).

[44] N. Cabrera, N. F. Mott, Theory of the oxidation of metals, Reports Prog. Phys. 12 (1) (1944) 163-184 (1944). arXiv:arXiv:1011.1669v3

[45] P. Pérez, V. A. C. Haanappel, M. F. Stroosnijder, The effect of niobium on the oxidation behaviour of titanium in N2/20\% O2 atmospheres, Mater. Sci. Eng. A 284 (1-2) (2000) 126-137 (2000).

[46] M. Delheusy, A. Stierle, N. Kasper, R. P. Kurta, A. Vlad, H. Dosch, C. Antoine, A. Resta, E. Lundgren, J. Andersen, X-ray investigation of subsurface interstitial oxygen at Nb/oxide interfaces, Appl. Phys. Lett. 92 (10) (2008).

[47] A. F. Carley, P. R. Chalker, J. C. Riviere, M. W. Roberts, The identification and characterisation of mixed oxidation states at oxidised titanium surfaces by analysis of X-ray photoelectron spectra, J. Chem. Soc. Faraday Trans. 183 (2) (1987) 351 (1987). 